\title{
Temporal frequency response characteristic of motion channels measured with three different psychophysical techniques
}

\author{
ALLAN J. PANTLE \\ Miami University, Oxford, Ohio 45056
}

\begin{abstract}
The temporal frequency response characteristic of motion-sensitive channels in human vision was studied with three different psychophysical techniques: (a) measurements of threshold flicker sensitivity to drifting sinusoidal gratings; (b) measurements of the amount of contrast possessed by one sinusoidal component of a composite adapting stimulus when it just nulled the motion aftereffect produced by a second reference component drifting in the opposite direction; and (c) measurements of velocity difference thresholds. The threshold flicker measurements (a) and null contrast measurements (b) corresponded exactly throughout the entire range of temporal frequencies studied as long as the velocity of the reference component in the null contrast method was not too fast. The correspondence means that the two types of measurements very likely have a common substrate comprised of directionally selective channels. The correspondence is also interesting in that one set of measurements (a) was obtained at threshold levels, whereas the second set of measurements (b) was obtained at suprathreshold levels. At high temporal frequencies, the function describing velocity difference thresholds departs from that which describes the other two types of measurements, suggesting that velocity discriminations at high temporal frequencies depend upon different cues or motion channels.
\end{abstract}

The goal of the present research was to isolate and describe the temporal response properties of motionsensitive mechanisms in the human visual system. To this end, three different psychophysical techniques were employed and the temporal frequency response characteristics measured with the different models were compared.

A number of investigators (e.g., Keesey, 1972; Kulikowski \& Tolhurst, 1973; Levinson \& Sekuler, 1975; Tolhurst, 1973) have pointed out that moving or flickering gratings have not one, but two distinct contrast thresholds. At one of the minimum contrast values, the movement or flicker of a grating is just visible. At another minimum contrast, the spatial structure of the grating becomes distinct. The separate thresholds have been termed the flicker threshold and the pattern-recognition threshold, respectively, and they are independent functions of the spatial and temporal frequency of gratings. ${ }^{1}$ Investigators who have taken the trouble to distinguish between the two

This research was partially supported by an Air Force contract, F33615-74-C-4032, through the Aerospace Medical Research Laboratory, Wright-Patterson Air Force Base, Ohio 45433. I thank Ken Shelley, Patty Smith, Liz Bauer-Nelson Sarders, Faith Steele, and Carol Formes, who served as subjects and/or helped with data collection and analysis for the various experiments. J.T. Petersik provided helpful comments for improving an earlier version of the manuscript. Finally, I wish to thank Jane Jackson for calling my attention to and ridding the manuscript of inconsistencies and grammatical errors as she typed it in its final form. types of threshold have done so largely because they believe that the separate thresholds represent activity in separate visual channels (e.g., Breitmeyer \& Ganz, 1976; Keesey, 1972; Kulikowski \& Tolhurst, 1973; Levinson \& Sekuler, 1975; Tolhurst, 1973). The flicker thresholds are assumed to reflect activity in motion-sensitive channels, or human analogues of transient neurons of the cat and monkey visual system (e.g., Enroth-Cugell \& Robson, 1966; Gouras, 1968), and the pattern-recognition thresholds are assumed to reflect activity in form-sensitive channels, or human analogues of sustained neurons. In a series of threshold, subthreshold summation, and selective adaptation experiments, Levinson and Sekuler (1975) have shown that the channels underlying flicker thresholds for single-component drifting gratings and counterphase gratings ${ }^{2}$ are directionally selective. In the case of the counterphase grating, independent directionally selective channels presumably respond to each of its oppositely moving components, and the flicker threshold is determined by whichever channel in momentarily the more sensitive. In our first experiment, we obtained flicker thresholds for a drifting sinusoidal grating and used them to define the temporal frequency response characteristic of directionally selective, motion channels.

One drawback of using flicker thresholds to estimate response properties of motion channels is that the procedure relies on a subject's ability to 
maintain a qualitative distinction between different kinds of threshold criteria. A second drawback is that the procedure can be used only with thresholdlevel signals. For this reason, we devised a psychophysical cancellation technique which we used to measure motion aftereffects at suprathreshold contrasts and which required a subject to act only as a null instrument.

The cancellation technique was developed in view of a number of empirical facts about motion aftereffects and their interpretation. After adaptation to a grating which moves uniformly in a single direction, a stationary grating will appear to move in the opposite direction (motion aftereffect or waterfall illusion). One popular interpretation of the aftereffect is that it is due to an imbalance in the response of directionally selective mechanisms (e.g., Sutherland, 1961). After adaptation, mechanisms tuned to the direction of the adapting motion are relatively less responsive than those tuned to other or the opposite direction of movement (e.g., Barlow \& Hill, 1963). The imbalance hypothesis is supported by an experiment by Sekuler and Ganz (1963), who found that, after adaptation to a moving grating, the visibility of a test grating moving in the same direction as the adapting grating was less than that of a test grating moving in the opposite direction. If the imbalance hypothesis is correct, then, consistent with an old finding, there should be no motion aftereffect after adaptation to a pattern which alternates between opposite directions of movement (Wohlgemuth, 1911). While two groups of directionally selective channels would be rendered less responsive after the adaptation, presumably each would be equally adapted and there would be no imbalance of their responses. Similarly, adaptation to a counterphase grating which contains two identical, simultaneous, oppositely moving components produces no motion aftereffect. In our second experiment, subjects adapted to a composite stimulus formed by the superposition of two simultaneous, oppositely moving sinusoidal components of unequal drift rates (temporal frequencies). By adjusting the contrast of one of the components, one whose temporal frequency was varied during the course of an experiment, it could be made to just cancel the aftereffect produced by the second component whose contrast and temporal frequency were kept constant. The cancelling contrast values of the variable component at each of a number of temporal frequencies were used to define a suprathreshold, constant-response function-a second type of temporal frequency characteristic for directionally selective, motion channels. It could be compared directly to that obtained by the flicker threshold method.

As a third measure of the temporal frequency response properties of directionally selective motion channels, we measured a subject's sensitivity to changes in the drift rate of moving sinusoidal gratings. In particular we determined (1) whether sensitivity to small changes in the drift rate $(\Delta \overline{D R})$ of a grating would vary as a function of its drift rate (DR) and, if so, (2) whether the relationship between $\triangle \overline{D R}$ and $\overline{\mathrm{DR}}$ would be similar to the temporal frequency characteristics measured by the flicker threshold method and the aftereffect cancellation method. Past research on sensitivity to changes of drift rate (e.g., Hick, 1950; Notterman \& Page, 1957) has been conducted only with nonperiodic stimuli whose description in the frequency domain is quite complex.

\section{GENERAL METHOD}

The same three subjects participated in all experiments. Two of the subjects (K.L.S. and P.L.S.) were university students, and the third subject (A.J.P.) was the author. Subject P.L.S. was naive with respect to the goals of the research. All subjects were emmetropic or wore correcting lenses to obtain 20/20 acuity.

In all experiments, the stimulus patterns were stationary sinusoidal gratings, drifting sinusoidal gratings, or spatiotemporal patterns formed by superimposing two sinusoidal drifting gratings. The patterns were displayed on the face of a Tektronix 5103 ocilloscope with a P31 phosphor. With minor modifications, the general technique described by Pantle (1971) was used to generate the stimulus gratings. In the motion aftereffect cancellation experiments, the spatiotemporal adapting pattern was th sum of two oppositely drifting sinusoidal gratings. In order to produce this pattern, two audio-oscillators were placed in the external circuit which supplied a complex modulating signal (the sum of two sinusoids) to the $\mathrm{Z}$-axis of the oscilloscope. The phase of one of the sinusoidal components of the modulating signal was continuously shifted in one direction relative to signal for the sweep generator of the scope, and the phase of the second component of the modulating signal was continuously shifted in the opposite direction. The subject viewed all displays through a rectangular opening in an illuminated cardboard surround. The patterns completely filled the opening and subtended a visual angle of $4^{\circ} 45^{\prime}$ horizontally and $4^{\circ} 06^{\prime}$ vertically. The cardboard surround was circular and its outside diameter was $10^{\circ} 53^{\prime}$. The luminance and color of the surround was matched to the space-average luminance $\left(5.7 \mathrm{~cd} / \mathrm{m}^{2}\right.$ ) and color (yellowgreen) of the oscilloscope patterns. The subject viewed the display binocularly with natural pupils from a distance of $107 \mathrm{~cm}$. A small black fixation spot was located in the center of the stimulus pattern.

\section{EXPERIMENT 1: THRESHOLD FLICKER SENSITIVITY}

In the first experiment, we obtained measurements of flicker or movement thresholds in the manner defined by Kulikowski and Tolhurst (1973). Their measurements were repeated with simple drifting gratings so that the temporal frequency response characteristics obtained by the aftereffect cancellation and the velocity $\overline{\mathrm{DL}}$ methods could be directly compared with flicker threshold data from the same subjects. Each subject adjusted the contrast of a 1-c/deg drifting sinusoidal grating until he could just detect the movement of the grating bars 
or the flicker produced by the drifting grating, whichever required the least contrast. Five contrast threshold measurements were obtained at each of nine drift rates (temporal frequencies between .3 and $20 \mathrm{~Hz}$ ). One measurement at each of the nine drift rates constituted a block, and the order of presentation of the drift rates was random within each block.

Contrast thresholds (log percent contrast as a function of the temporal drift rate) ${ }^{3}$ of the grating are shown in Figure 1. Each function represents the data for a different subject. Each data point is the mean of the five threshold estimates obtained for each subject at a given drift rate. The standard errors of the means plotted in Figure 1 ranged from .01 to .06 with a mean of .03 (about $7 \%$ ). As reported in similar past studies, the contrast threshold for flicker or movement is lowest at an intermediate temporal frequency and increases at both higher and lower temporal frequencies.

\section{EXPERIMENT 2: CANCELLATION TECHNIQUE FOR MEASURING MOTION AFTEREFFECT}

The motion aftereffect cancellation technique exploits the basic waterfall illusion-after an observer views contours moving uniformly in one direction

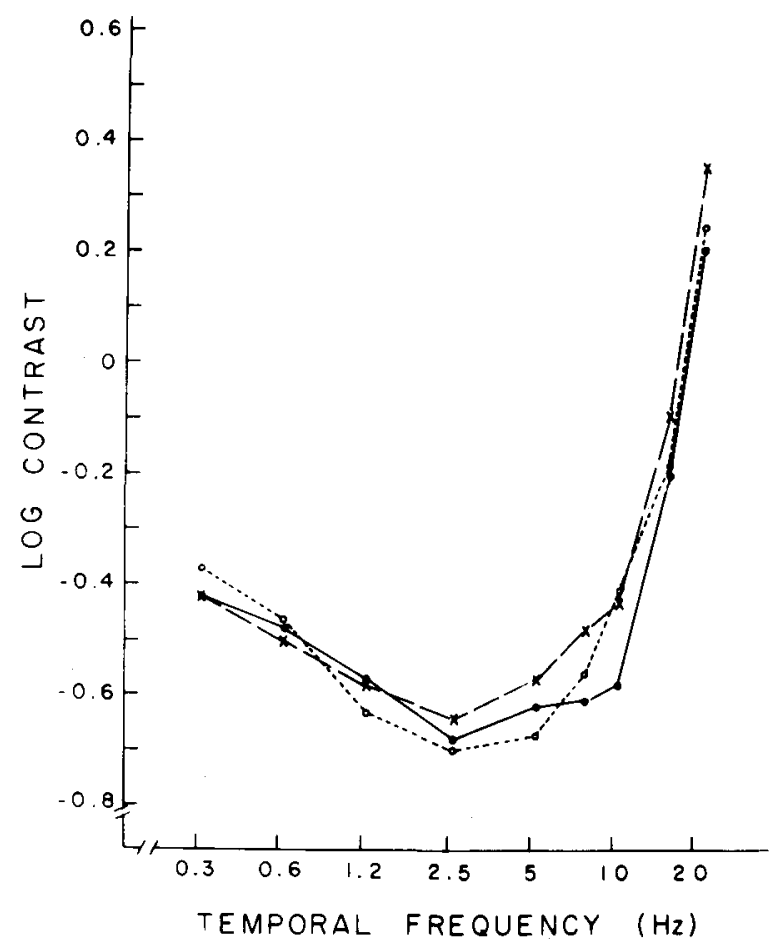

Figure 1. Contrast thresholds for detecting the movement or flicker of drifting sinusoidal gratings as a function of their temporal drift rate. Observer P.L.S., @ s; observer A.J.P., X s; and observer K.L.S., Os. for a prolonged period of time, stationary contours will appear to move in the opposite direction. In Experiment 2, a motion aftereffect was generated by having subjects adapt to a $1-\mathrm{c} / \mathrm{deg}$ sinusoidal grating which moved rightward (hereafter this grating will be referred to as $f_{R}$ ). A stationary sinusoidal test grating $(1 \mathrm{c} / \mathrm{deg})$ that was presented afterwards appeared to move to the left. The adapting and test patterns alternated, $8 \mathrm{sec}$ for the adapting stimulus and $2 \mathrm{sec}$ for the test stimulus. The repetitive sequence allowed the subject to see a strongraftereffect a number of times. Presumably, what happens under these conditions according to the imbalance hypothesis of the motion aftereffect is that the rightward-moving adapting grating reduces the responsiveness of a set of rightward-sensitive mechanisms and thereby produces an imbalance in the responsiveness of leftward- and rightwardsensitive mechanisms. The imbalance, in turn, results in the apparent leftward motion of the test grating. By adding a leftward moving component to the adapting stimulus, it should be possible to adapt leftward-sensitive mechanisms as well as the rightward-sensitive mechanisms, and thereby eliminate the imbalance which produces the aftereffect.

After the first few adapting-test cycles (trials) in any session, a second sinusoidal adapting grating $(1 \mathrm{c} / \mathrm{deg})$ was superimposed upon $\mathrm{f}_{\mathrm{R}}$ during the adaptation period. The second adapting grating (hereafter called $f_{L}$ ) moved to the left. Indeed, by adjusting the contrast of $f_{L}$, a subject could cancel or null the motion aftereffect produced by $f_{R}$. For low contrasts of $f_{L}$, the aftereffect generated by $f_{R}$ was only weakened; at high contrasts of $f_{L}$, the aftereffect generated by $f_{R}$ was overridden and reversed; but at some intermediate contrast (hereafter called the null contrast), the aftereffect generated by $f_{R}$ was just cancelled (i.e., the interspersed test grating appeared stationary). At the null contrast, leftward- and rightward-sensitive mechanisms would be adapted equally according to the imbalance hypothesis.

The determination of the null contrast for any given $f_{L}$ required a series of adjustments over a number of trials. Initially, the contrast of $f_{L}$ was set well below the null contrast at a randomly determined starting point. Thereafter, the subject increased or decreased the contrast of $f_{L}$, depending upon the direction and magnitude of the aftereffect he saw on each trial. At each contrast level of $f_{L}$, a few trials were required for the adaptation process underlying the aftereffect to reach a steady state. Especially when the contrast of $f_{L}$ was in the vicinity of the null contrast, the subject waited for a number of trials before making a further change in the contrast of $f_{L}$. The subject terminated the adjustment series when he was satisfied that he saw 
no aftereffect during the test period. The contrast of $f_{L}$ at that time provided one estimate of the null contrast. Each adjustment series typically lasted 10-15 min.

Three independent estimates of the null contrast were obtained at each of nine different drift rates (temporal frequencies) of $f_{L}$. The nine drift rates were arranged in blocks, and the order of presentation within a block was random. Variations of the null contrast as a function of the drift rate of $f_{L}$, for a fixed contrast and drift rate of $f_{R}$, define a suprathreshold temporal frequency response characteristic for motion-sensitive channels. The characteristic is a constant-response function, since it describes changes of input (null contrast) required to produce a constant output, i.e., a constant amount of adaptation produced by $f_{L}$ to cancel a standard (fixed) directional aftereffect produced by $f_{R}$.

Three separate frequency response functions were obtained, one for each of three drift rates of $f_{R}$. For each function, the drift rate of $f_{R}$ was held constant at $1.25,5$, or $15 \mathrm{~Hz}$. At each drift rate, the contrast of $f_{R}$ was also held constant at one of the values given in Table 1 . The physical contrasts were approximately 10 times the subjects' flicker thresholds. The actual factor is given in parentheses behind the physical contrast. The test grating contrast used for each subject is also given in Table 1. The test contrasts were approximately 10 times the subjects' pattern thresholds for the test grating. ${ }^{4}$

Results obtained with a 5-Hz drift rate of $f_{R}$ are shown in Figure 2. The abcissa is the temporal drift rate of $f_{L}$. The ordinate is the null contrast, i.e., the relative contrast of $\mathrm{f}_{\mathrm{L}}$ required to cancel the aftereffect produced by $f_{R}$. It is expressed as the logarithm of the ratio of the contrast of $f_{L}$ to $f_{R}$. If the null contrast for a given frequency of $f_{L}$ were equal to the contrast of $f_{R}$, then the ratio of $f_{L}$ to $f_{R}$ would be one and the logarithm of the ratio would be zero and lie on the dashed line. If the null contrast were greater than that of $f_{R}$, then the data point would fall above the dashed line. If the null contrast were less than that of $f_{R}$, then the data point would fall below the dashed line. Each data point is the mean of the three estimates

Table 1

Contrast Values Used for Each of a Number of Different Rates of $\mathbf{f}_{\mathbf{R}}$ for Each Subject to Obtain a Temporal Frequency Response Function

\begin{tabular}{lcccc}
\hline & \multicolumn{3}{c}{ Drift Rate of $\mathbf{f}_{\mathbf{R}}$} & $\begin{array}{c}\text { Test } \\
\text { Grating }\end{array}$ \\
\cline { 2 - 5 } Observer & 1.25 & 5 & 15 & $.62(11 \mathrm{X})$ \\
\hline P.L.S. & $.23(11 \mathrm{X})$ & $.26(11 \mathrm{X})$ & $.64(10 \mathrm{X})$ \\
A.J.P. & $.26(11 \mathrm{X})$ & $.19(7 \mathrm{X})$ & $.51(7 \mathrm{X})$ & $.46(10 \mathrm{X})$ \\
K.L.S. & $.21(11 \mathrm{X})$ & $.23(11 \mathrm{X})$ & $.73(11 \mathrm{X})$ & $.59(10 \mathrm{X})$ \\
\hline
\end{tabular}

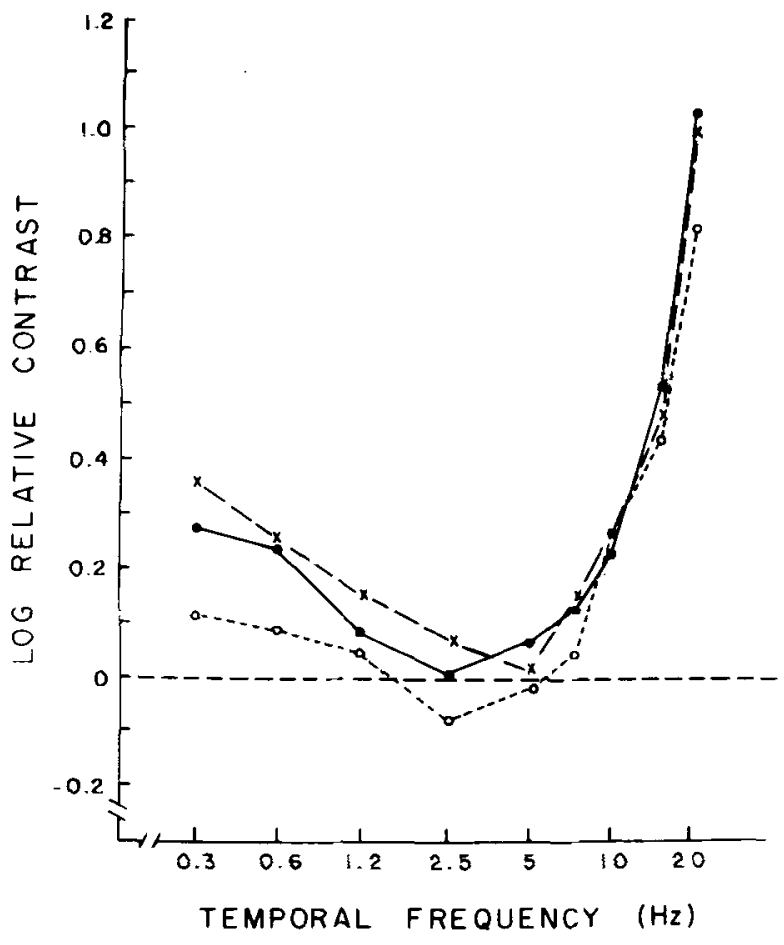

Figure 2. Relative null contrast required for one adapting component $\left(f_{L}\right)$ to cancel the aftereffect generated by a second adapting component $\left(f_{R}\right)$ drifting at $5 \mathrm{~Hz}$ as a function of the drift rate (temporal frequency) of $f_{L}$. Observer P.L.S., os; observer A.J.P., X s; observer K.L.S., Os.

of the null contrast obtained for each condition. The average standard error of the mean was .035 $(\mathbf{8 \%})$. Each function represents the data obtained for a different subject. The functions for the three subjects are essentially the same. In order to cancel the aftereffect generated by $f_{R}$ when it drifted at $5 \mathrm{~Hz}$, the subject adjusted the contrast of $f_{L}$ when it drifted at $5 \mathrm{~Hz}$ to approximately the same value (within experimental error) as that of $f_{R}$. (For each subject, the data point lies close to the dashed line.) The relative null contrast increased for lower temporal frequencies of $f_{L}(<1.25 \mathrm{~Hz})$ and for high temporal frequencies of $f_{L}(>5 \mathrm{~Hz})$, and it was at a minimum in the vicinity of $2.5-5 \mathrm{~Hz}$. This means that the aftereffect generated by $f_{R}$ was most readily cancelled by $f_{L}$ when $f_{L}$ moved at a drift rate of approximately $2.5-5 \mathrm{~Hz}$. Other temporal frequencies were less effective. The same pattern of results was obtained when the drift rate of $\mathrm{I}_{R}$ was $1.25 \mathrm{~Hz}$ (see Figure 3). The relative null contrast was minimal at $2.5-5 \mathrm{~Hz}$ and increased at both higher and lower temporal frequencies. The temporal frequency response functions for the $1.25-$ and $5-\mathrm{Hz}$ drift rates of $f_{R}$ are remarkably similar in shape to the flicker thresholds functions shown in Figure 1 for the same subjects. The temporal frequency response functions obtained with an $\mathrm{f}_{\mathrm{R}}$ drift rate of $15 \mathrm{~Hz}$ are shown in 


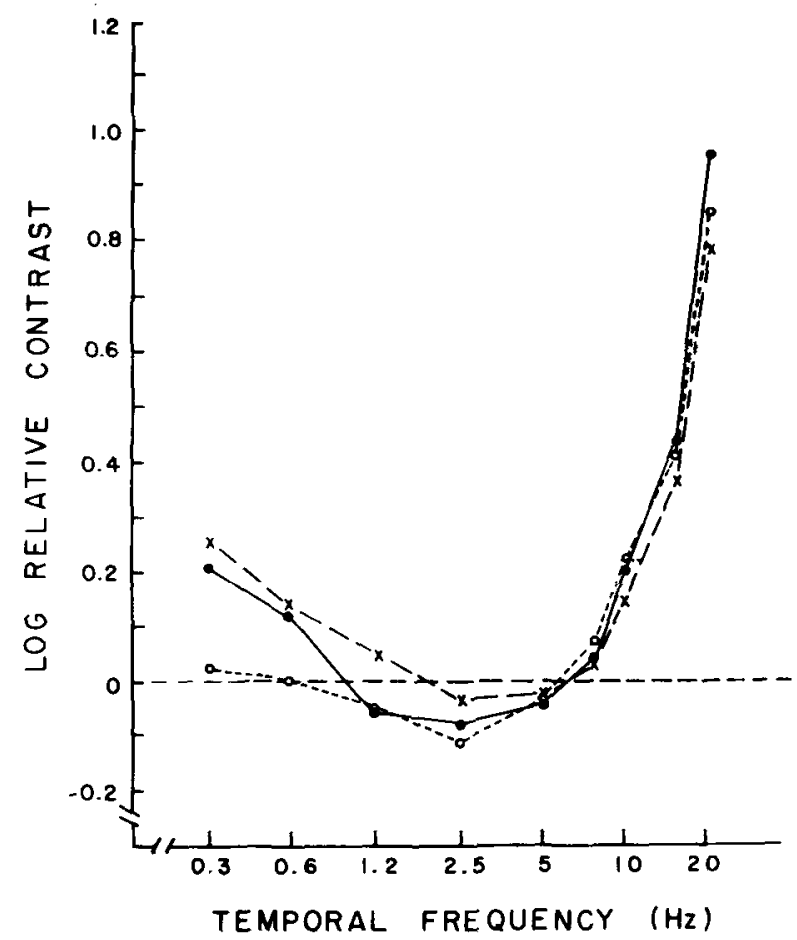

Figure 3. Relative null contrast required for one adapting component $\left(f_{L}\right)$ to cancel the aftereffect generated by a second adapting component $\left(f_{R}\right)$ drifting at $1.25 \mathrm{~Hz}$ as a function of the drift rate (temporal frequency) of $f_{L}$. Observer P.L.S., os; observer A.J.P., X s; observer K.L.S., Os.

Figure 4. They are different in shape from the functions obtained with $f_{R}$ drift rates of 1.25 and $5 \mathrm{~Hz}$. They have a low-pass characteristic; the least null contrast was required at low temporal frequencies of $f_{L}$, and in general it increased with the temporal frequency of $f_{L}$.

\section{EXPERIMENT 3: VELOCITY DIFFERENCE THRESHOLDS}

The method of constant stimuli was used to measure the ability of a subject to discriminate differences of drift rates of sinusoidal gratings. A trial consisted of two discrete temporal intervals. A grating moving rightward at one of five preselected test velocities was presented in the first interval. A grating moving leftward at a standard velocity was presented in the second interval. Two of the test velocities were faster, two were slower, and one was equal to the standard velocity. In order to avoid sharp temporal transients when the gratings were switched "on" and "off," the contrast of the grating presented in each interval was increased linearly from 0 to .20 during the first $250 \mathrm{msec}$ of the interval, was held at a plateau of .20 for $2,000 \mathrm{msec}$, and then was decreased linearly from .20 to 0 during the last $250 \mathrm{msec}$. The period between stimulus intervals was $1,500 \mathrm{msec}$. After each trial, the subject indicated whether the standard velocity was faster or slower than the test velocity. The time between trials was typically about 3-4 sec. Each test velocity was presented 30 times in a random order with the constraint that no test velocity was presented the $n^{\text {th }}$ time before all the test velocities had been presented $n-1$ times. The velocity difference threshold ( $\overline{\mathrm{DL}})$ was derived from the function which related proportion of "slower" judgments to test velocity. The velocity $\overline{\mathrm{DL}}$ was defined as one-half the difference between the test velocities that yielded $\mathbf{7 5 \%}$ and $25 \%$ "slower" judgments. In most cases, simple graphic interpolation (Klings \& Riggs, 1972, pp. 25-26) was employed to determine the test velocities which corresponded to the $75 \%$ and $25 \%$ points. In cases when the function relating proportion of "slower" judgments to test velocity was nonmonotonic, or when none of the test velocities yielded proportions of "slower" judgments either greater than $75 \%$ or less than $25 \%$, the proportions were first converted to $z$ scores and then a leastsquares method (Klings \& Riggs, 1972, pp: 27-29) was used to determine the test velocities corresponding to the $75 \%$ and $25 \%$ points.

Velocity $\overline{\mathrm{DL}} \mathrm{s}$ were obtained for a number of standard velocities ranging from .3 to $20 \mathrm{~Hz}$. The standard velocities were presented in a nonsystematic

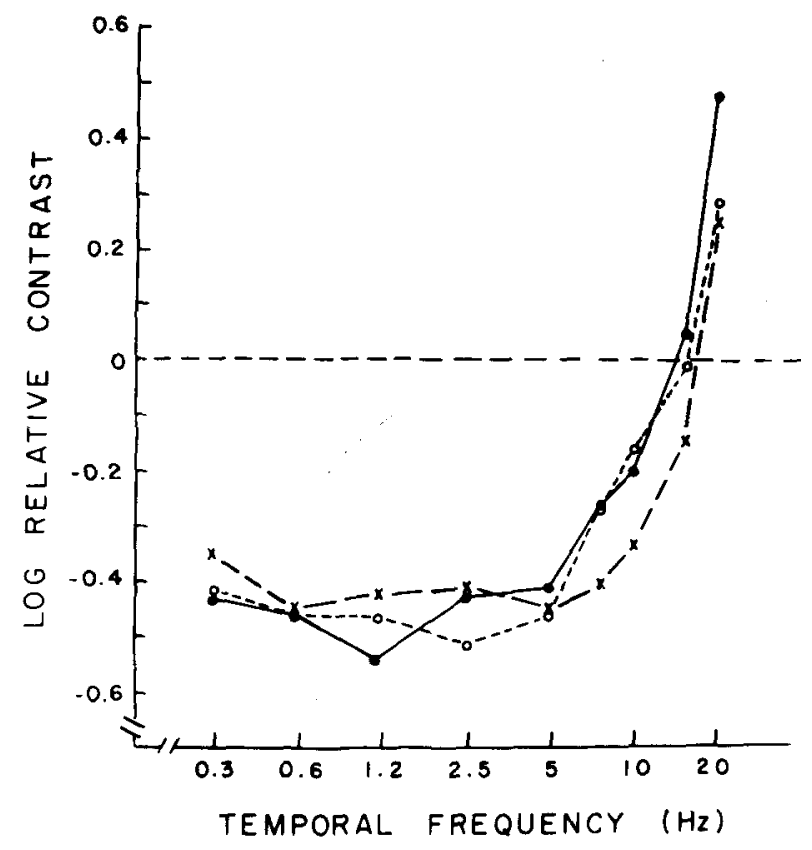

Figure 4. Relative null contrast required for one adapting component $\left(f_{L}\right)$ to cancel the aftereffect generated by a second adapting component $\left(f_{R}\right)$ drifting at $15 \mathrm{~Hz}$ as a function of the drift rate (temporal frequency) of $f_{L}$. Observer P.L.S., os; observer A.J.P., X s; observer K.L.S., Os. 
order. Measurements of DLs were obtained with gratings of three different spatial frequencies in the following order: $4.8, .6$, and $10 \mathrm{c} / \mathrm{deg}$. The viewing distance for the .6-c/deg gratings was changed to $53 \mathrm{~cm}$. At this distance, the visual angles subtended by the parts of the stimulus display were approximately a factor of two greater than those described earlier.

The results of Experiment 3 are shown in Figure 5. In each panel, the velocity $\overline{D L}$ (expressed as a percent of the drift rate of the standard) is plotted as a function of the drift rate of the standard. Each panel gives the results obtained with a different spatial frequency. Each function in each panel describes the results for a different subject. A comparison of the vertical positions of the functions across the panels of the figure shows the effect of spatial frequency on the subject's ability to detect velocity differences. The vertical position of the functions shifts upward from the left to the right panel in the figure, showing that the velocity DLs increased as spatial frequency was increased from .6 to $10 \mathrm{c} / \mathrm{deg}$. An analysis of variance of the data shown in Figure 5 indicates that the main effect of spatial frequency is statistically significant $[F(2,4)$ $=98.1, \mathrm{p}<.0002]$. The main effect of drift rate is also statistically significant $[F(7,14)=173.5$, $p<.0001]$, and the effect can be seen more clearly in Figure 6. Each function in Figure 6 is the average of the three individual functions in one of the panels in Figure 5, i.e., the average function for a particular spatial frequency. The relationship between the velocity $\overline{D L}$ and drift rate was essentially the same for all spatial frequencies. Figure 6 shows that velocity discrimination improved (DLs decreased) as drift rate was increased from .5 to $2.5-5 \mathrm{~Hz}$, grew slightly worse as drift rate was

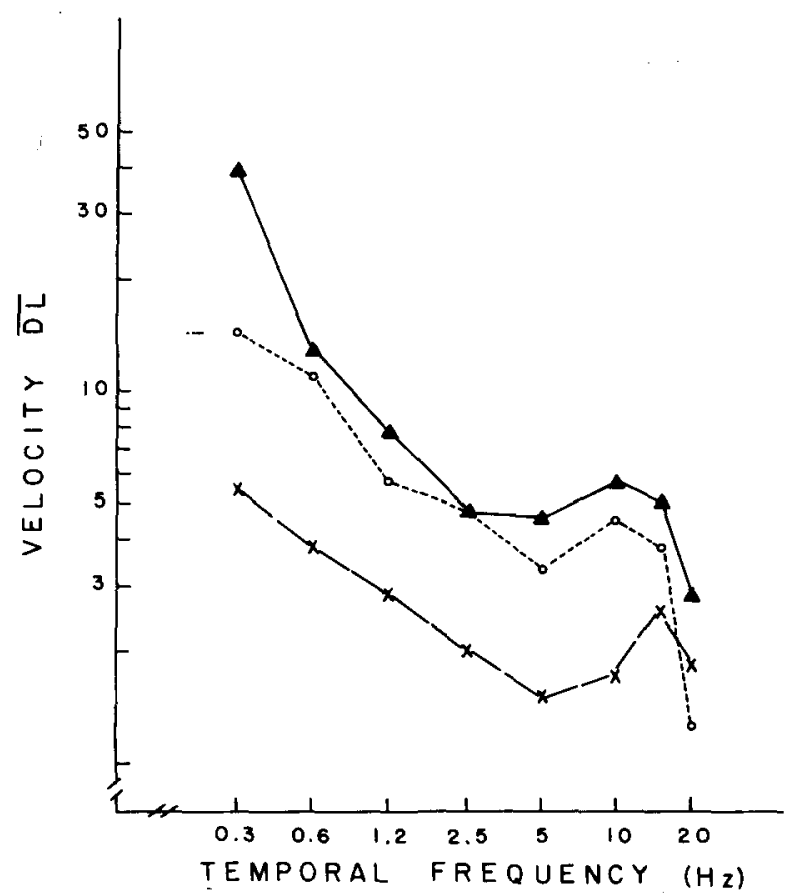

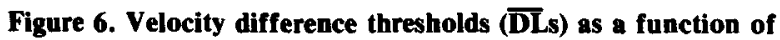
the temporal frequency of sinusoidal gratings. Data depicted with Xs, open circles, and triangles were obtained with $.6-, 4.8-$, and $10-c / d e g$ gratings, respectively.

increased further to about $10-15 \mathrm{~Hz}$, and then improved again for a drift rate of $20 \mathrm{~Hz}$.

\section{DISCUSSION}

\section{Comparisons Among the Temporal Frequency}

Response Characteristics of Experiments 1, 2, and 3

Temporal frequency characteristics obtained with the three different psychophysical methods are

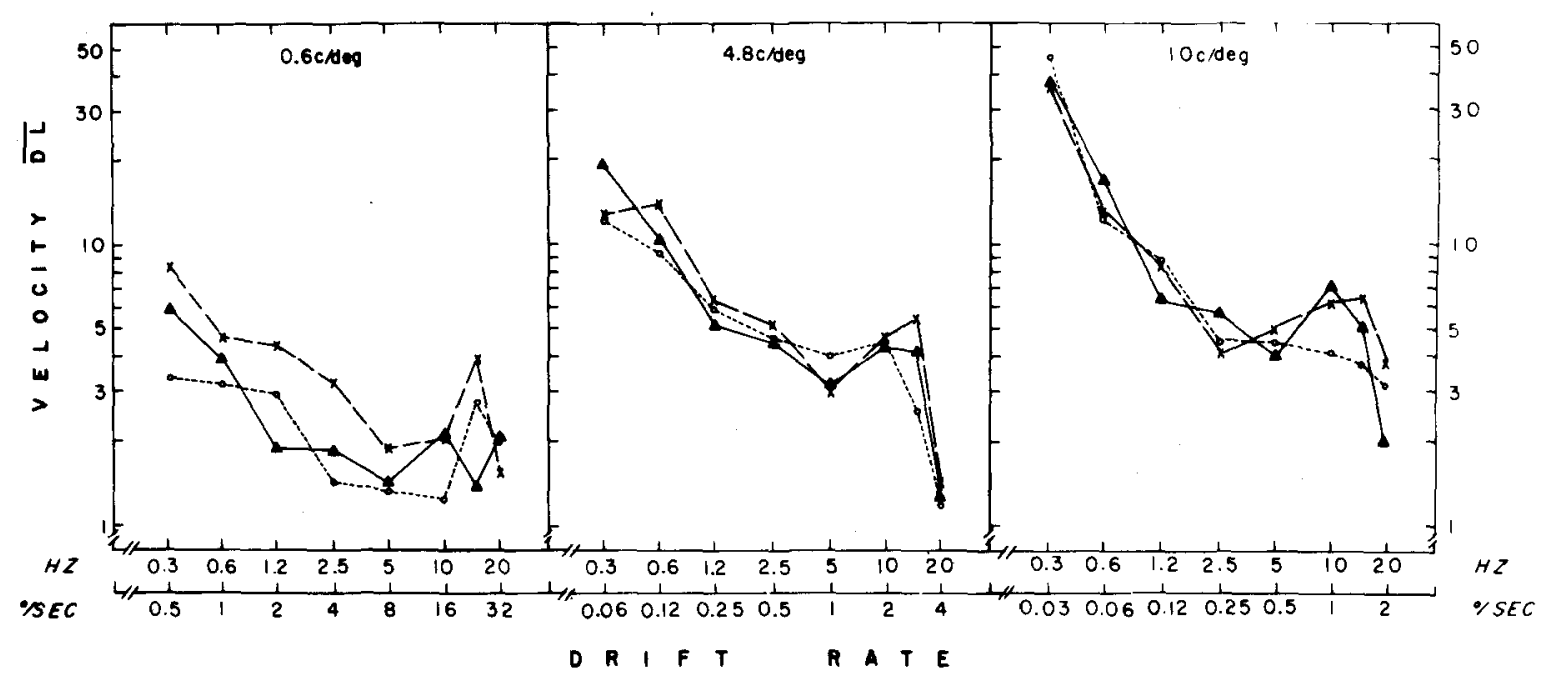

Figure 5. Velocity difference thresholds ( $\overline{\mathrm{DL}} \mathrm{s}$ ) as a function of the drift rate of sinusoidal gratings. Observer P.L.S., Xs; observer A.J.P., $\Delta$ s; observer K.L.S., Os. 
summarized in Figure 7 . The function drawn with the solid line is a summary of the flicker threshold measurements of Figure 1. The mean of the flicker thresholds of the individual subjects (Figure 1) was found at each temporal frequency. The mean thresholds were then normalized to give a maximum sensitivity (minimum threshold) of zero, and the normalized data are plotted in Figure 7. Smaller values indicate greater sensitiviy, and sensivity increases from the bottom to the top of the graph. As can be seen in the figure, flicker sensitivity is greatest at $2.5 \mathrm{~Hz}$ and decreases at both higher and lower temporal frequencies.

The function drawn with short dashes in Figure 7 summarizes the aftereffect cancellation data of Figure 2. The mean of the null contrasts of the leftward-moving grating $\left(f_{L}\right)$ which were required by the individual observers to cancel the aftereffect generated by the rightward-moving grating $\left(f_{R}\right)$ moving at $5 \mathrm{~Hz}$ was computed for each drift rate of $f_{L}$. The means were normalized to give a minimim null contrast of zero, and the normalized null contrasts are plotted in Figure 7 as the short-dash function. As can be seen in Figure 7, the null contrast function is identical to the temporal frequency characteristic obtained by the flicker threshold method. The null contrast function has a maximum of $2.5 \mathrm{~Hz}$, and it falls off at the same rate as the flicker threshold function at lower and higher temporal frequencies. The shapes of the null

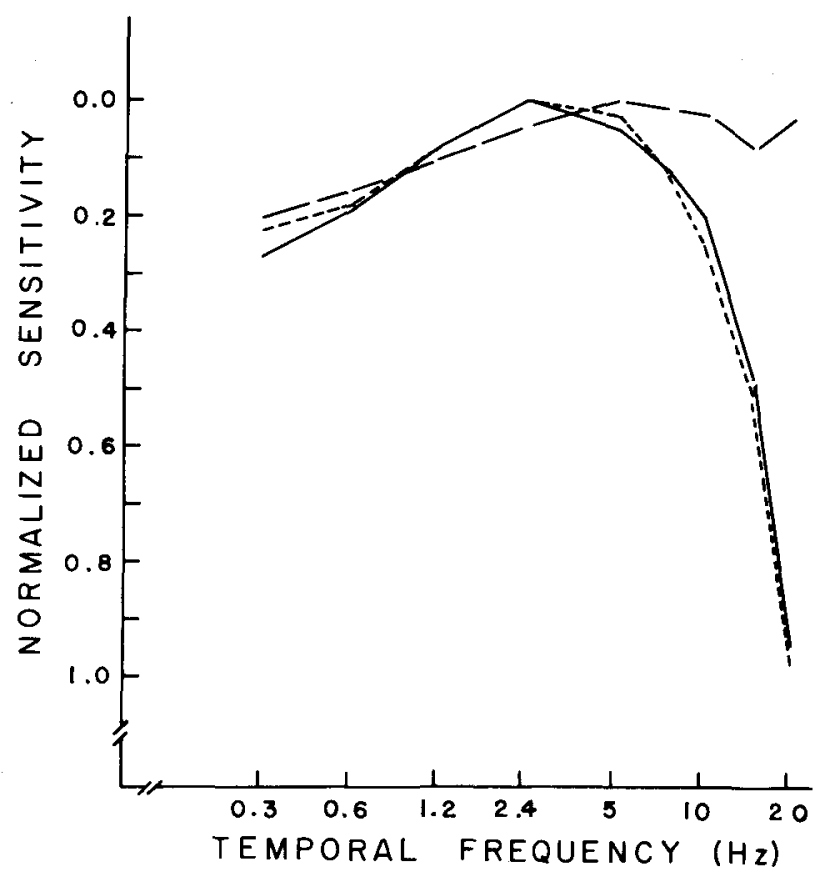

Figure 7. Temporal frequency response characteristics derived from measurements of threshold flicker sensitivity (solid line), measurements of motion aftereffect cancellations (short dashes), and measurements of velocity difference thresholds (long dashes). contrast functions obtained with a standard drift rate of $1.25 \mathrm{~Hz}$ (Figure 3) are essentially the same as the shapes obtained with the standard drift rate of $5 \mathrm{~Hz}$ (Figure 2). Thus, it does not matter which of the two sets of data are compared to the temporal frequency characteristic obtained by the flicker threshold method. The null contrast function obtained with the $1.25-\mathrm{Hz}$ drift rate matches the temporal frequency characteristic obtained by the flicker threshold method as well as the $5-\mathrm{Hz}$ null contrast function. However, the shapes of the null contrast functions obtained with the standard drift rate of $15 \mathrm{~Hz}$ (Figure 4) were different from those of the null contrast functions for standard drift rates of 1.25 and $5 \mathrm{~Hz}$. It may be that a drift rate of $15 \mathrm{~Hz}$ approaches the temporal resolution limit of the directionally selective mechanisms which underlie the motion aftereffect. For this reason, the $15-\mathrm{Hz}$ null contrast data were not used for the comparisons in Figure 7, and they do not enter into the theoretical conclusions discussed in a later section.

The function drawn with the long dashes in Figure 7 was derived from the mean DL data represented by the $\mathrm{Xs}$ in Figure 6. These DL data were obtained with the .6-c/deg grating, which was closest in spatial frequency to the 1-c/deg gratings used in Experiments 1 and 2. The mean DLs were converted to logarithms, and the logarithms were scaled such that (1) the minimim $\overline{D L}$ received a value of zero and (2) the deviations of the scaled DL values from the average of the nuil contrast and flicker sensitivity data in the .3-to-2.5-Hz region were minimized. It is clear from Figure 7 that velocity discrimination is like threshold flicker sensitivity and aftereffect sensitivity in that it has a low-frequency falloff and peaks in the $2.5-5-\mathrm{Hz}$ region, but that it is unlike the other functions in that it does not exhibit a pronounced decline at high temporal frequencies.

\section{Theoretical Implications}

The temporal frequency characteristic which we obtained with the flicker threshold method and drifting sinusoidal gratings has the same shape as those obtained in past research (Kulikowski \& Tolhurst, 1973) with counterphase and on-off sinusoidal gratings. In simple studies of this type, there is no compelling reason to believe that the thresholds are mediated by transient channels which are directionally selective. However, after Levinson and Sekuler (1975) conducted studies in which they (1) compared the threshold contrasts of singlecomponent (simple sinusoidal) moving gratings and counterphase gratings, (2) described changes in the appearance of a counterphase grating after adaptation to a unidirectional moving grating, and (3) measured changes in the threshold for a counterphase grating when superimposed upon drifting subthreshold sinusoidal backgrounds both before and 
after adaptation to a unidirectional moving grating, they concluded that flicker thresholds for simple drifting sinusoidal gratings and for counterphase gratings were mediated by directionally selective; transient channels.

If the imbalance hypothesis of the motion aftereffect is correct, then the null contrast functions of Experiment 2 must also reflect response characteristics of directionally selective, transient channels. At each temporal frequency of $f_{L}$, a given amount of adaptation had to be produced in leftwardsensitive (directionally selective) mechanisms to compensate for the constant amount of adaptation produced by the fixed $f_{R}$ on rightward-sensitive mechanisms. One might argue that the null contrast functions are peculiar in some way and apply only to the adaptation properties of the directionally selective mechanisms, but the nearly exact correspondence of the null contrast functions and the flicker threshold functions of Experiment 1 suggests otherwise, namely that both types of functions reflect some type of general sensitivity or responsiveness of directionally selective mechanisms. This point is made especially clear if one considers one general implication of the correspondence between the two types of functions. If one wanted to cancel the motion aftereffect produced by a sinusoidal grating drifting at any rate (within limits) and having an amplitude of $\mathrm{X}$ times its flicker threshold, all one need do is to add a sinusoidal grating drifting in a direction opposite the first and having an amplitude of $\mathrm{X}$ times its own flicker threshold. The prediction would hold for any drift rate of the added component.

In a series of threshold and adaptation experiments with composite gratings containing leftward- and rightward-moving components, Sekuler and Levinson (1974) concluded that different laws of sensory behavior applied to directionally selective mechanisms at threshold and at suprathreshold levels of stimulation. At threshold levels, they claim that the responses of directionally selective mechanisms tend to be independent, whereas at suprathreshold levels, they tend to interact or inhibit one another. Certainly, our aftereffect cancellation experiment permitted inhibitory interactions between two oppositely tuned, directionally selective mechanisms responding at suprathreshold levels, whereas the flicker threshold experiment involved at most only one set of directionally selective mechanisms responding at threshold levels. How can the measured behavior in the two experiments be so similar if different laws of behavior apply to the stimuli in the two experiments? One possibility is that the cancellation experiments do, indeed, involve inhibitory interactions, but that the cancellation procedure precludes the possibility of seeing them. We believe this possibility to be true. Figure 8 is adapted from the simple motion model

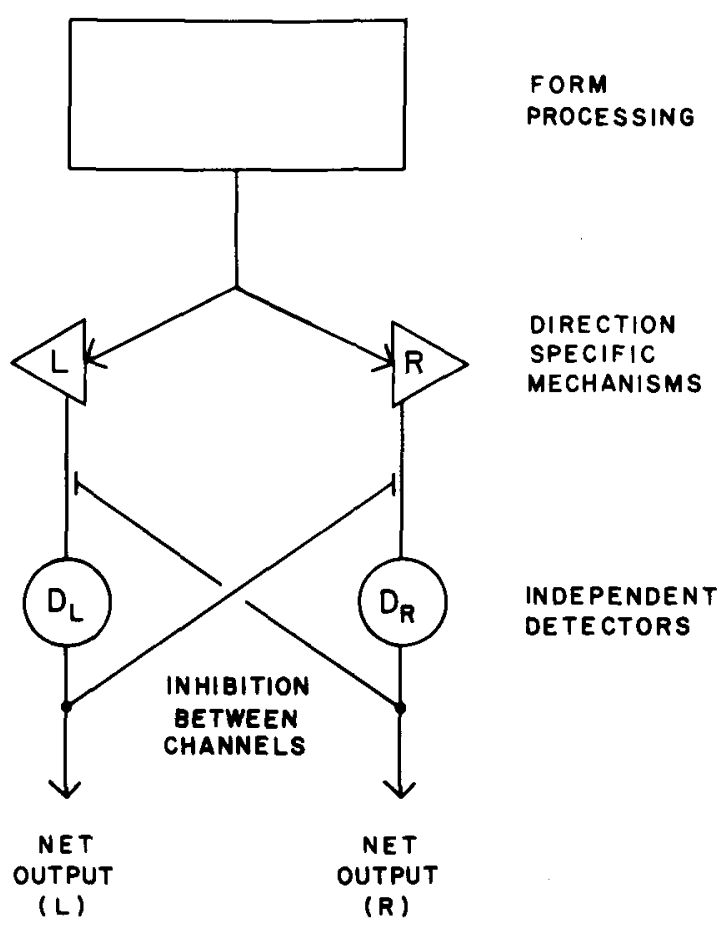

Figure 8. A simple model of some aspects of motion perception in human vision. Adapted from Sekuler and Levinson (1974).

depicted in Figure 6 of Sekuler and Levinson (1974), and the model is consistent with a wide variety of experiments which they conducted. The first stage of the model involves some preprocessing of the form of the stimulus. The next stage consists of separate (parallel) directionally selective mechanisms. For the sake of simplicity, only leftward- and rightwardsensitive mechanisms are represented. The output of each directionally selective mechanism goes to a separate detector, permitting the channels to act independently at threshold. Once the activity in either channel exceeds some threshold amount, it exerts a recurrent inhibitory effect on the opposite channel.

Now consider what presumably happens in our aftereffect cancellation experiments at the null contrast setting according to the Sekuler and Levinson model. The standard component $\left(f_{R}\right)$ of the composite adapting stimulus excites the rightward-sensitive channel, which in turn produces a recurrent inhibitory effect on the leftward-sensitive channel. When the contrast of $f_{L}$ is set to the null point, there will be enough excitation in the leftward-sensitive channel and enough concomitant inhibition flowing to the rightward-sensitive channel, such that the net response in each channel is equal and there is equal adaptation of each channel. At each of the different temporal frequencies of $f_{L}$, different null contrasts of $f_{L}$ are required to equalize the adaptation in each channel. But, because $f_{R}$ remains constant for any null contrast function, the excitation in the leftward- and rightwardsensitive channels and the inhibition flowing in either 
direction between them would remain unchanged in magnitude whatever the frequency of $f_{L}$ or whatever its null contrast. As a consequence, the cancellation procedure is, in effect, blind to the inhibitory interactions between channels because they never vary in magnitude for the different temporal frequencies of $f_{L}$.

The close correspondence between the shapes of the suprathreshold null contrast functions and the threshold flicker functions suggests that directionally selective neurons behave in a linear fashion. The linear behavior may seem difficult to reconcile with past research which indicates that the response of directionally selective mechanisms saturates at low contrasts, i.e., at about 5-6 times threshold (Pantle, Lehmkuhle, \& Caudill, 1978), The reconciliation is achieved without much difficulty. (1) The response saturation described in the Pantle et al. paper was obtained with single-component gratings. In the aftereffect cancellation experiments, the mutual inhibition between channels which is caused by the two simultaneous adaptation components, even though they are 10 times their individually measured thresholds, may keep the net response in each channel below the response saturation level. (2) It is important to emphasize that the flicker threshold functions are constant-output functions; i.e., some criterion output must be reached for a grating to be at threshold at a number of temporal frequencies. Also, as alluded to before, null contrast functions are constantoutput functions; i.e., some criterion amount of adaptation must be produced by $f_{L}$ at a number of temporal frequencies to compensate for a standard (fixed) amount of adaptation produced by $f_{R}$ in an oppositely tuned set of directionally selective mechanisms. The criterion outputs which must be achieved in either case are simply different points on the output function of directionally selective mechanisms, and neither the flicker threshold experiment nor the aftereffect cancellation experiment alone says anything about the exact functional relationship among various inputs and outputs. Different outputs were not measured in either experiment.

It is possible that measurements of threshold flicker sensitivity and aftereffect sensitivity reflect the number of transient channels or elements tuned to different spatial or temporal frequencies, with sensitivity being directly related to the concentration of tuned channels or elements at a particular frequency. If so, velocity $\overline{\mathrm{DL}}$ s might be expected to mirror flicker and aftereffect sensitivity because a small change of the velocity of a stimulus to which many transient channels are tuned would involve a change in a large number of responding channels. ${ }^{5}$ It is interesting, then, that velocity $\overline{\mathrm{D} L S}$ increase monotonically with spatial frequency (Figure 5 or Figure 6) as flicker thresholds have been shown to do (Kulikowski \& Tolhurst, 1973).
The difficulty with attributing the velocity discrimination performance to directionally selective transient channels is that the $\overline{\mathrm{DL}}$ function does not exhibit a pronounced decline at high temporal frequencies as do the threshold flicker sensitivity and the aftereffect sensitivity functions. The basis of velocity discrimination at high temporal frequencies must be different from that of threshold flicker sensitivity and the motion aftereffect. The different basis can be interpreted in more than one way. For example, velocity discriminations may depend upon perceived tifferences in drift rate per se (associated with differences in the output of transient channels) at low and intermediate temporal frequencies, but upon other cues such as perceived contrast or blur at high temporal frequencies. ${ }^{6}$ Or, it is possible that velocity discriminations at high temporal frequencies depend upon a qualitatively different type of flicker-sensitive mechanism. In a flicker adaptation experiment, Pantle (1971) found that mechanisms which responded to the 3- and $32-\mathrm{Hz}$ flicker of a uniform field could each be selectively adapted. The $32-\mathrm{Hz}$ channel might be very sensitive to changes of temporal frequency in the vicinity of its preferred frequency (i.e., 20-35- $\mathrm{Hz}$ region), yet it may not prevent the decline of threshold flicker sensitivity at high temporal frequencies because it has a high contrast threshold or contribute to motion aftereffects because its response is not direction-specific.

\section{REFERENCES}

Barlow, H. B., \& HrLl, R. M. Evidence for a physiological explanation of the waterfall phenomenon and figural aftereffects. Nature, 1963, 200, 1345-1347.

BreItMEYER, B. G., \& GANz, L. Implications of sustained and transient channels for theories of visual pattern masking, saccadic suppression, and information processing. Psychological Review, 1976, 83, 1-36.

Enroth-Cugell, C., \& Robson, J. G. The contrast sensitivity of retinal ganglion cells of the cat. Journal of Physiology, $1966,187,517-552$.

Gouras, $P$. Identification of cone mechanisms in monkey ganglion cells. Journal of Physiology, 1968, 199, 533-547.

Hick, W. E. The threshold for sudden changes in the velocity of a seen object. Quarterly Journal of Experimental Psychology, $1950,2,33-41$.

KEESEY, U. Flicker and pattern detection: A comparison of thresholds. Journal of the Optical Society of America, 1972, 62, 446-448.

Kling, J. W., \& Rigcs, L. A. (Eds.). Woodworth \& Schlosberg's experimental psychology (Vol. 1). New York: Holt, Rinehart and Winston, 1972.

Kulikowski, J. J., \& Tolmurst, D. J. Psychophysical evidence for sustained and transient detectors in human vision. Journal of Physiology, 1973, 232, 149-162.

Levinson, E., \& SEkuler, R. The independence of channels in human vision selective for direction of movement. Journal of Physiology, 1975, 250, 347-366.

Notterman, J. M., \& Page, D. E. Weber's law and the difference threshold for the velocity of a seen object. Science, $1957,126,652$. 
Pantle, A. Flicker adaptation-1. Effect on visual sensitivity to temporal fluctuations of light intensity. Vision Research, 1971, 11, 943-952.

Pantle, A., Lehmuxuhle, S., \& Caudill, M. On the capacity of directionally selective mechanisms to encode different dimensions of moving stimuli. Perception, 1978, 7, 261-267.

Sexuler, R. W., \& Ganz, L. Aftereffect of seen motion with a stabilized retinal image. Science, 1963, 139, 419-420.

SeKuler. R.. \& LeVinson, E. Mechanisms of motion perception. Psychologia, 1974, 17, 38-49.

Sutherland, N. S. Figural aftereffects and apparent size. Quarterly Joumal of Experimental Psychology, 1961, 13, 222-228.

TOLHURST, D. J. Separate channels for the analysis of the shape and the movement of a moving visual stimulus. Journal of Physiology, 1973, 231, 385-402.

WoHLGEMUTH, A. On the after-effect of seen movement. British Journal of Psychology Monographs, Supplement 1, 1911.

\section{NOTES}

1. Because the two thresholds are independent functions of spatial and temporal frequency, the appearance of a temporally modulated grating which is just detectable can be quite different. At the detection threshold, the grating will look like a spatially uniform field or like a series of more or less formless ripples if the flicker threshold of the grating is lower than the patternrecognition threshold. The grating will be indistinguishable from an identical stationary pattern at the detection threshold, if its pattern-recognition threshold is lower than its flicker threshold.

2. A counterphase grating is a grating with a sinusoidal luminance profile whose dark and light stripes reverse repetitively in time. It can be decomposed into two oppositely moving, equal-amplitude, sinusoidal components of the same spatial and temporal frequency.
3. The temporal drift rate or temporal frequency (hertz) of a grating is equal to its spatial frequency (cycles/degree) multiplied by its velocity (degrees/second). Since the spatial frequency of the grating in Experiments 1 and 2 was $1 \mathrm{c} / \mathrm{deg}$, the temporal frequency of the grating represented by the abcissae of Figures 1-4 is numerically equal to the velocity of the grating.

4. The absolute values of the contrast of $f_{R}$ and of the test grating are provided for the sake of completeness only. The exact values are not critical for the results, and findings are expressed as relative or normalized contrasts.

5 . The proposed hypothesis about the relationship between the three psychophysical measures and the concentration of transient cells is only one of a number of possible alternatives which could be advanced in view of the paucity of data which shows how the three measures vary under common stimulus conditions. As an alternative, it seems possible to imagine a scheme whereby velocity $\overline{\mathrm{DL}} s$ would be maximal at intermediate velocities where flicker sensitivity changes little with velocity and would be small at high velocities where flicker sensitivity changes considerably with velocity. As a second alternative, the flicker sensitivity measurements may depend only upon the output of the single channel or channels most sensitive to a given stimulus and the velocity $\overline{\mathrm{DL}}$ measurements upon a comparison of the relative outputs of all channels stimulated by a given stimulus.

6. Supplementary experiments in which the contrast of the test grating of Experiment 3 was varied randomly over a range of .5 log unit and independently of its velocity had no measureable effect on the magnitude of the velocity $\overline{\mathrm{DL}} \mathrm{s}$. This means that subjects probably did not use changes in perceived contrast as a velocity cue at high temporal frequencies.

(Received for publication April 25, 1978; revision accepted July 17,1978 .) 